\title{
From the Editors
}

It has been 5 years since the ill-fated oil tanker, Exxon Valdez, ran aground, spilling its cargo of Alaskan crude into Prince William Sound. The resulting pollution was so diffuse and profound that despite years of cleanup the damaged ecosystem is still struggling to recover. There is current evidence that our ethical "ecosystem" is also vulnerable to "Exxon Valdez"-type disasters.

New and shocking revelations regarding past radiation experiments at major American universities and Department of Energy laboratories have become almost daily fare in the mass media. Similarly, secret bacteriological tests were inflicted on unwitting populations throughout the Cold War. One almost forgotten exercise, carried out within close range of $C Q$ 's editorial office, involved technicians aboard the USS ACM-13, a few miles offshore, spraying San Francisco with bacterialaden aerosol bombs for 6 days. Citizens were hospitalized with "mysterious" lung infections, and one man died. Add to this the downwind effects on populations subjected to atomic bomb testing after World War II, and one wonders just how close American government research came to paralleling Nazi experimentation. Nor is the United States the only government to engage in such research activities without participant consent.

Simultaneously with the exposé of earlier widespread and flagrant problems, an escalating number of contemporary violations are being reported in the media. For example, from 1982 to 1990 no more than a handful of reports involving research ethics were covered in the press. In 1991, only one infraction was reported. By 1993, however, there were reports of eight separate incidences, and in the first quarter of 1994, five different reports had already appeared in addition to the widely reported radiation experiments. With respect to numbers of cases reported to the Office of Protection from Research Risk at the National Institutes of Health, since 1990 there have been 169 investigations into research infractions, 115 of which have been completed. Some of these are serious infractions leading, for example, to the suspension of the breast cancer study for falsification of data in 1994.

Ghosts from our past then, coupled with an increasing preponderance of current reports, create the alarming perception of research ethics run amuck. How are we to respond? Without trying to soft-pedal our failures, one could argue that the system of checks and balances on research ethics is working. There are many indications that some of the self-correcting mechanisms in the system that are supposed to work, in fact, have been brought into play - even though some were initiated after the fact and proved to be an example of too little too late. One question that emerges from research ethics reports is to what extent should earlier infractions be held to higher and later standards? 
There also have been institutional lapses in the protection of research subjects, for example, in the area of continuing review. It is ironic that in the field of science, where data are so important, we spend an inordinate amount of time reviewing the hypothesis and look less at the experiences and the findings. Although the "up front" protection of subjects is carefully planned, clearly anticipating and avoiding problems, what actually transpires in carrying out the research is not examined seriously enough. Another lapse involves a thorough understanding on the part of Institutional Review Boards (IRBs) and investigators regarding the rights of research subjects and their protection. There are also lapses when procedures sometimes escape review; the most grievous are cases where researchers simply opt themselves out of the review process. Much more difficult to evaluate are medical and surgical procedures that are on the cutting edge of therapies. Some people take the position that it is acceptable to move forward and improvise in these situations, the argument being that the rules governing research do not apply because what is being done is not really research but rather "innovative therapy."

Lapses, such as those described above, point to flaws in a system that is believed to be adequate but sometimes does not work very well. A thorough systematic review of the performance of the IRB system is now underway, directed by Charles MacKay, under the auspices of the National Institutes of Health. Results and recommendations are expected in early 1995. However, in fairness, it should be acknowledged that IRBs are facing new and increasing pressures; they are caught in the changing tide of expanding activity. As a result, the job is sometimes beyond the work capacity of the members of IRBs, as they are currently constituted. For example, since the mid-1970s the work- load for IRBs has at least tripled, whole new areas of research have begun (e.g., AIDS), greater monitoring of approved protocols is required, cooperative studies have increased between institutions and drug companies, research increasingly involves women and minorities who are at risk, and troubling research protocols using placebos are on the rise.

These challenges are coupled with a new widespread attitude. The familiar emphasis on the burden side of the riskbenefit ratio is shifting. Instead, research is increasingly seen as a benefit to which everyone ought to have access. The possibilities of what research may offer subjects, especially the most seriously ill patients, have created the view that perhaps there is a "right" to participate. Following this view, rather than judgments on appropriateness made by an invisible and paternalistic board, patient autonomy alone is supported by some as the proper regulating force for whether or not people should join a research protocol.

All the resulting furor over past mistakes and our failure to have adequate corrective procedures in place, whether in response to oil spills or research atrocities, cannot repair the dreadful harms that have been done in the name of science and progress. This fact in no way diminishes the importance of heeding the wake-up call that these painful events provide. A wake-up call should not, however, lead to a precipitous rush toward a quick fix that eventually evaporates with very little residual effect. We need longer range perspectives that require our willingness to 1 ) take the time to look again at our methods and procedures, because clearly when they become routinized and taken for granted we invite disaster; 2) adopt new attitudes that elevate the value of human experience and make an understanding of the needs of others a paramount objective of all medical research; and 3) develop new procedures to meet cur- 
rent challenges, especially those arising from the medical-industrial complex of drug companies, genetic corporations, healthcare corporations, and medical research.

The papers in this issue's Special Section, "Research Ethics: Ethics at the Borders of Medical Research," call for a redirection of our energy and commitment on these three levels. The shared assumption uniting these papers, rang- ing from responsible conduct in scientific research regarding children, women, and the cognitively impaired elderly, to the relationship between science and society, including the internal influences of IRBs, is that a wounded ethical landscape can and should serve the useful purpose of making us look at prospective protection with a new degree of resolve. We invite your concern and response. 